The rod is then screwed to the end of the tube of the injection apparatus by means of the screw point in which is a fiber washer to make the joint tight. The rod is then clamped in a mechanical pipette holder, either that of Barber or one described in an article already printed. The next step is to fill the pipette with mercury. To do this open the stopcock and see that the rubber tubing connected with the stopcock is full of mercury. With a strong clamp close the tubing about four inches from the stopcock. Along this four inches place several screw clamps which, on being screwed down, will produce sufficient pressure to drive mercury almost to the tip of the pipette. The stopcock is then to be securely shut off.

We are now ready for action. Squeezing the metal tubes by one or other of the leverage clamps will drive mercury through a pipette having an aperture of only one micron $(.001 \mathrm{~mm}$.) in diameter. Move the pipette by means of the pipette holder till its tip projects into a hanging drop of the solution to be injected. Release pressure on the steel tube and some of the solution will be drawn into the pipette. Now lower the pipette and move the moist chamber till the cell to be injected is brought into view. The pipette is now raised until it punctures the cell. On applying pressure to the steel tube the solution is readily injected. The apparatus may also be used to withdraw materials from the cell.

The apparatus is extraordinarily sensitive. The meniscus of the mercury in the pipette responds instantly to the pressure of the leverage clamps. A comparative estimation of the quantity of injection material used may be made by focusing, first, on the mercury meniscus, then on the tip of the pipette and measuring the distance of the two focal points by means of the fine adjustment screw of the microscope.

A more complete description of this apparatus will shortly be published.

\section{ROBert Chambers}

Cornell Medical College
ON THE EMISSION AND ABSORPTION OF OXYGEN AND AIR IN THE EXTREME ULTRA-VIOLET

UP to this time very little has been known of the spectrum of oxpgen in the region of wave-lengths shorter than $\lambda 2000$. Some previous investigators were unable to obtain a spectrum in this region. "No lines or bands," says Lyman, "were observed between $\lambda 2000$ and 1230." 1 Schumann, however, had succeeded in photographing some continuous maxima of which the most refrangible has a wave-length of about 1850 Angstroms. Moreover, Lyman had observed that the great absorption band of oxygen diminishes in intensity as it approaches $\lambda 1230$, but he thinks that another absorption band exists "lying in the region shut out by the absorption of fluorite." This preliminary investigation was undertaken, therefore, to test the emission and absorption of oxygen and air in the region of wave-lengths shorter than those transmitted by fluorite.

The apparatus used consisted of a vacuum grating spectrograph, containing a Rowland concave grating of 50 centimeters focus, about 15,000 lines per inch, and a ruled surface of approximately 2 inches. A discharge tube of internal capillary, end-on type and with aluminum electrodes was employed. The tube was also provided with a quartz window for $\mathrm{Hg}$ comparison spectrum and opened through a slit directly into the receiver. A method has been developed of making Schumann films, and these were used for the spectrograms. Commercial oxygen, dried with phosphorus pentoxide, filled the receiver and connected discharge tube to a pressure of about $0.4 \mathrm{~mm}$. When the spectrum of air was obtained, this gas was likewise dried and filled the receiver to about the above pressure. The time of exposure varied from 20 minutes to 2 hours for the gas spectra, while an exposure of 3 minutes was found to be suffcient for the $\mathrm{Hg}$-arc comparison spectrum. The apparatus was so arranged that both the first and second orders of the Lyman region

1 Lyman, "The Spectroscopy of the Extreme Ultra-violet,', p. 82. 
appeared on the film, the second order being superimposed on the first order comparison spectrum. By the use of the foregoing method, an extensive spectrum was obtained with oxygen in the receiver and is attributed to that gas. A spectrum was also found for air. (See table.)

\section{TABLE}

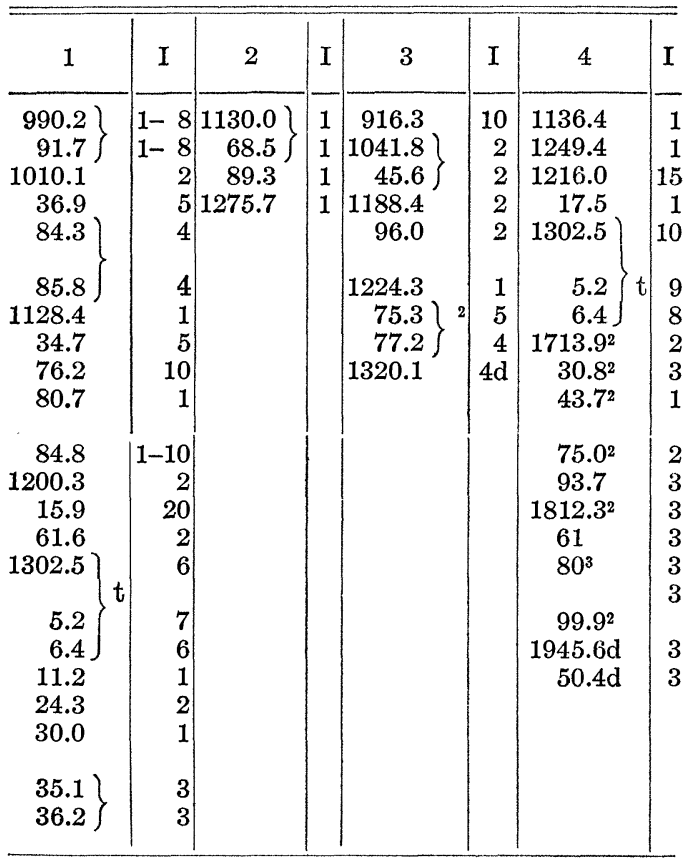

Explanation of Symbols of Table: \} indicates doublet; $\}$ triplet; $\} 1$ doublet and probably triplet; $d$ diffuse; 2 violet edge of band; 3 continuous maximum.

In almost every instance the wave-lengths given in the table are the averages of two or more plates, hence judging from the consistency of the several measurements they are believed to be accurate to about 0.5 Angstrom. The first column contains the lines that occur in both air and oxygen. Column 2 gives the lines that were not registered on the films of air spectra, but were on those of the spectrum of oxygen. They are faint. Some of the more intense lines that occur in air only are indicated in column 3; the fainter lines, of which there are about thirty, were omitted from the list. Column
4 is a record of the wave lengths produced in oxygen with direct current discharge. The spectra listed in columns 1, 2 and 3 were obtained with disruptive discharge. In column 4 , lines in the Schumann region are included; similar spectra were also present in the cases of disruptive discharge but were omitted from the tabulations.

The other columns are lists of the relative intensities of the wave-lengths in the columns immediately preceding. Where two values of intensity are given in the same column, the first refers to the spectrum with oxygen and the second with air.

It is worthy of note that the line $\lambda 1215.9$ is very strong in the spectrum of oxygen and air even when a direct current was used. This wave length is very near to $\lambda 1215.6$, the fundamental line in the hydrogen spectrum, and probably is that line. This was found to be present in most of the spectra obtained by Millikan in his investigations on the spectra of metals. The transparency of oxygen and air (1340-916 for air and 1336990 for oxygen) in this region is proved from the fact that these spectrograms were obtained. It is evident that the absorbing layer of gas in these experiments amounted to more than $0.5 \mathrm{~mm}$. at atmospheric pressure, and judging from the intensities of the spectra, these gases are transparent in layers of even much greater thickness. The films of the spectrum of air were badly fogged, and in some cases the entire spectrum appeared reversed. However, since other films of this spectrum were obtained without this reversal, it is believed to be of a chemical nature and due to the corrosive gases formed by the radiation or the discharge. This point will be investigated more thoroughly in the near future. The work of getting the spectra of electrolytic oxygen and pure nitrogen is now on the way, and the thorough search for series lines and for ionization and resonance potential relations is postponed until this new data is available.

\section{J. J. HOPFIELD}

Departuent of Physics, University of California 